University of Florence

DFF-343/8/99

\title{
Effective Theory for Color-Flavor Locking in High Density QCD
}

\author{
R. Casalbuoni ${ }^{a, b}$ and R. Gatto ${ }^{c}$ \\ ${ }^{a}$ Dipartimento di Fisica, Università di Firenze, I-50125 Firenze, Italia \\ ${ }^{b}$ I.N.F.N., Sezione di Firenze, I-50125 Firenze, Italia \\ ${ }^{c}$ Départ. de Physique Théorique, Université de Genève, CH-1211 Genève 4, \\ Suisse
}

\begin{abstract}
We describe the low energy excitations of the diquark condensates in the color-flavor locked phase of QCD with three massless flavors, at high baryon densities, in terms of a non-linear effective lagrangian. Such a lagrangian is formally seen to correspond to the lagrangian for the hidden gauge symmetry description of the effective low energy chiral lagrangian, the role of the hidden symmetry being played by color. In particular, this agrees with the conjecture that the light degrees of freedom of the two phases are in correspondence to each other (complementarity). The discussion includes consideration of the breaking of Lorentz invariance in presence of matter density.
\end{abstract}




\section{Introduction}

We introduce an effective lagrangian describing the low energy excitations of the diquark condensates in the color-flavor locked (CFL) phase of QCD with three massless flavors at high chemical potential. It is expected that this phase is complementary to the low density chirality breaking phase by identification of the light degrees of freedom in the two phases. Within our effective description it is straightforward to formally proof this statement by showing that the effective color-gauged lagrangian in the CFL phase is the non-linear lagrangian for the low density chirality breaking phase with explicit couplings to the octet of vector resonances. In fact we find that the effective lagrangian in the CFL phase corresponds exactly to the socalled hidden gauge symmetry description of the effective low energy chiral lagrangian with the role of the hidden symmetry played by the (non hidden) gauge symmetry. The breaking of Lorentz invariance in presence of matter density does not hinder this conclusion. The effective description we present here for color-flavor locking constitutes a simplified description of the full QCD dynamics. More quantitative details may only come from a complete dynamical treatment within QCD. Our approach exhibits however the main features of the color-locking phenomenon and it may be useful in view of its simplicity.

Understanding the behaviour of QCD in the domain of high densities and small temperatures is expected to be important for the study of neutron stars and hopefully also for heavy ion collisions which are studied in different high energy laboratories. Early studies [1] had suggested that a new phenomenon, color superconductivity, would take place in those conditions.

Particular attention has been given to the study of QCD with three massless flavors at high values of the baryonic chemical potential and zero temperature. At very high chemical potential one expects calculations based on one-gluon exchange to be justified to derive the main traits of the superconductive dynamics. For this case the condensation pattern would imply the phenomenon of color-flavor locking [2]. In addition to the implied breaking of chiral symmetry in the locked phase, gluons are expected to become massive and an interesting correspondence takes place between the spectrum of the low lying states of the color-locked phase and the hadronic spectrum in the chirally broken phase at low chemical potential [3]. This feature (complementarity) will be a main interest in the present note. 
Dynamical calculations of the superconductive gap have been carried out by different authors [4]. Beyond the ideal case of QCD with three massless flavors, dynamical studies have also been attempted for two massless flavors $(\mathrm{u}, \mathrm{d})$ and a massive s-quark, in the same region of high chemical potential. The construction we present here can easily be modified to cover such a situation for the case of finite strange quark mass and sufficiently large chemical potential. Also the more complicated situations where both quark-antiquark and diquark condensates are present have been examined. The extension of our effective lagrangian to such a possible situation is still feasible although more cumbersome.

\section{The effective theory}

The CFL phase is characterized by the symmetry breaking pattern

$$
G=S U(3)_{c} \otimes S U(3)_{L} \otimes S U(3)_{R} \otimes U(1) \rightarrow H=S U(3)_{c+L+R}
$$

due to the dynamical formation of condensates of the type

$$
\left\langle\psi_{a i}^{L} \psi_{b j}^{L}\right\rangle=-\left\langle\psi_{a i}^{R} \psi_{b j}^{R}\right\rangle=\kappa_{1} \delta_{a i} \delta_{b j}+\kappa_{2} \delta_{a j} \delta_{b i}
$$

where $\psi_{a i}^{L(R)}$ are Weyl spinors and a sum over spinor indices is understood. The indices $a, b$ and $i, j$ refer to $S U(3)_{c}$ and to $S U(3)_{L}$ (or $S U(3)_{R}$ ) respectively.

We are interested in building up a low energy effective description of the CFL phase, that is, roughly, for momenta which are small with respect to the energy gap characterizing this phase (the numerical estimates so far give values between 10 and $100 \mathrm{MeV}$ for the gap energy). The decay coupling constants of the Goldstones are expected to be of the same order of magnitude of the gap. In order to describe this breaking pattern we need 17 Goldstone fields (for the moment being we will consider only global symmetries and soon after come back to the gauging of color).

We introduce two types of coset fields transforming with respect to $G$ respectively as a left-handed and a right-handed quark field. By denoting these fields by $X$ and $Y$ we require

$$
X \rightarrow g_{c} X g_{L}^{T}, \quad Y \rightarrow g_{c} Y g_{R}^{T}
$$


with

$$
g_{c} \in S U(3)_{c}, \quad g_{L} \in S U(3)_{L}, \quad g_{R} \in S U(3)_{R}
$$

with $X$ and $Y$ being $S U(3)$ matrices breaking respectively $S U(3)_{c} \otimes S U(3)_{L}$ and $S U(3)_{c} \otimes S U(3)_{R}$. This gives us 16 Goldstone fields. We then need an additional one to describe the breaking of the baryon number. This is done in terms of a $U(1)$ factor transforming under $G$ as

$$
U \rightarrow g_{U(1)} U, \quad g_{U(1)} \in U(1)
$$

For the following it is convenient to define the following currents

$$
J_{X}^{\mu}=X \partial^{\mu} X^{\dagger}, \quad J_{Y}^{\mu}=Y \partial^{\mu} Y^{\dagger}, \quad J_{\phi}=U \partial^{\mu} U^{\dagger}
$$

enjoying the following transformation properties with respect to the global group $G$

$$
J_{X}^{\mu} \rightarrow g_{c} J_{X}^{\mu} g_{c}^{\dagger}, \quad J_{Y}^{\mu} \rightarrow g_{c} J_{Y}^{\mu} g_{c}^{\dagger}, \quad J_{\phi}^{\mu} \rightarrow J_{\phi}^{\mu}
$$

Notice that all these currents are anti-hermitian and furthermore, since $J_{X}^{\mu}$ and $J_{Y}^{\mu}$ belong to the Lie algebra of $S U(3)$, they are traceless. Then, barring WZW terms [5] (they are necessary for the completeness of the theory but we will not discuss them here, except for a comment at the end), the most general Lorentz invariant lagrangian invariant under $G$, with at most two derivatives, is given by

$$
\mathcal{L}=-a \operatorname{Tr}\left[J_{X}^{\mu} J_{X \mu}\right]-a \operatorname{Tr}\left[J_{Y}^{\mu} J_{Y \mu}\right]-2 c \operatorname{Tr}\left[J_{X}^{\mu} J_{Y \mu}^{\dagger}\right]-d J_{\phi}^{\mu} J_{\phi \mu}
$$

At this point we leave sub judice the question of Lorentz invariance which does not hold in presence of matter density. We shall come back soon after to this point.

Notice that the term proportional to $c$ is essential, otherwise this lagrangian would be more symmetric than necessary, since it would be invariant under $G \otimes S U(3)$. In fact, one could act with independent transformations on the color indices of the matrices $X$ and $Y$. It is just this term which allows to identify the color groups acting upon these two fields. Such kind of term is lacking in ref. [6]. Also we have required the theory to be invariant under parity, that is we require symmetry under the exchange $X \leftrightarrow Y$.

For the following it will be useful to write the previous lagrangian in the form

$$
\mathcal{L}=-\frac{F^{2}}{4} \operatorname{Tr}\left[\left(J_{X}^{\mu}-J_{Y}^{\mu}\right)^{2}\right]-\alpha \frac{F^{2}}{4} \operatorname{Tr}\left[\left(J_{X}^{\mu}+J_{Y}^{\mu}\right)^{2}\right]-\frac{f^{2}}{2}\left(J_{\phi}^{\mu}\right)^{2}
$$


where we have defined the parameters

$$
a-c=\frac{F^{2}}{2}, \quad a+c=\alpha \frac{F^{2}}{2}, \quad d=\frac{f^{2}}{2}
$$

Let us now define the Goldstone canonical fields. We start with

$$
X=e^{i \tilde{\Pi}_{X}^{a} T_{a}}, \quad Y=e^{i \tilde{\Pi}_{Y}^{a} T_{a}}, \quad U=e^{i \tilde{\phi}}, \quad a=1, \cdots 8
$$

and $T_{a}$ the generators of $S U(3)$ with the following normalization (they are the Gell-Mann matrices up to a factor)

$$
\operatorname{Tr}\left[T_{a} T_{b}\right]=\frac{1}{2} \delta_{a b}
$$

By defining

$$
\begin{aligned}
\Pi_{X} & =\sqrt{\alpha} \frac{F}{2}\left(\tilde{\Pi}_{X}+\tilde{\Pi}_{Y}\right) \\
\Pi_{Y} & =\frac{F}{2}\left(\tilde{\Pi}_{X}-\tilde{\Pi}_{Y}\right) \\
\phi & =f \tilde{\phi}
\end{aligned}
$$

we get at the lowest order in the Goldstone fields the properly normalized kinetic term

$$
\mathcal{L}_{\text {kin }}=\frac{1}{2} \partial_{\mu} \Pi_{X}^{a} \partial^{\mu} \Pi_{X}^{a}+\frac{1}{2} \partial_{\mu} \Pi_{Y}^{a} \partial^{\mu} \Pi_{Y}^{a}+\frac{1}{2} \partial_{\mu} \phi \partial^{\mu} \phi
$$

Therefore the expression of the original fields in terms of the kinetic eigenstates is given by

$$
\begin{aligned}
X & =\exp \left(\frac{i}{F}\left(\frac{1}{\sqrt{\alpha}} \Pi_{X}^{a}+\Pi_{Y}^{a}\right) T_{a}\right) \\
Y & =\exp \left(\frac{i}{F}\left(\frac{1}{\sqrt{\alpha}} \Pi_{X}^{a}-\Pi_{Y}^{a}\right) T_{a}\right) \\
U & =\exp \left(\frac{i}{f} \phi\right)
\end{aligned}
$$


Let us now discuss the breaking of Lorentz invariance in presence of a matter density. We have to treat differently time and spatial derivatives. In the lagrangian (8) we should make substitutions of the type

$$
a \operatorname{Tr}\left[J_{X}^{\mu} J_{\mu X}^{\dagger}\right] \rightarrow a_{T} \operatorname{Tr}\left[J_{X}^{0} J_{0 X}^{\dagger}\right]+a_{S} \operatorname{Tr}\left[J_{X}^{i} J_{i X}^{\dagger}\right]
$$

since we still have rotational invariance. The canonical term for the Goldstone bosons is then

$$
\begin{aligned}
\mathcal{L}_{\mathrm{kin}} & =\frac{1}{2} \dot{\Pi}_{X}^{a} \dot{\Pi}_{X}^{a}+\frac{1}{2} \dot{\Pi}_{Y}^{a} \dot{\Pi}_{Y}^{a}+\frac{1}{2} \dot{\phi} \dot{\phi} \\
& -\frac{1}{2} v_{X}^{2} \vec{\nabla} \Pi_{X}^{a} \cdot \vec{\nabla} \Pi_{X}^{a}-\frac{1}{2} v_{Y}^{2} \vec{\nabla} \Pi_{Y}^{a} \cdot \vec{\nabla} \Pi_{Y}^{a}-\frac{1}{2} v_{\phi}^{2} \vec{\nabla} \phi \cdot \vec{\nabla} \phi
\end{aligned}
$$

where, with obvious notations

$$
v_{X}^{2}=\frac{\alpha_{S} F_{S}^{2}}{\alpha_{T} F_{T}^{2}}, \quad v_{Y}^{2}=\frac{F_{S}^{2}}{F_{T}^{2}}, \quad v_{\phi}^{2}=\frac{f_{S}^{2}}{f_{T}^{2}}
$$

Therefore the three different types of Goldstone bosons move with different velocities, but still satisfying a linear dispersion relation $E=v p$. Of course, since we expect here all the dimensioned quantities to be of the order of the gap, we expect also the velocities to be of order 1 . However, since 8 of these Goldstone bosons must be eaten up by the gluons, we will discuss again this point at the end of the next Section.

\section{The gauging of $S U(3)_{c}$}

The lagrangian of eq. (9) must have a local $S U(3)_{c}$ invariance inherited by the color group of QCD. In order to make this invariance explicit we need only to substitute the usual derivatives with covariant ones

$\partial_{\mu} X \rightarrow D_{\mu} X=\partial_{\mu} X-g_{\mu} X, \quad \partial_{\mu} Y \rightarrow D_{\mu} Y=\partial_{\mu} Y-g_{\mu} Y, \quad g_{\mu} \in$ Lie $S U(3)_{c}$

The corresponding currents are given by

$$
J_{X}^{\mu}=X \partial^{\mu} X^{\dagger}+g^{\mu}, \quad J_{Y}^{\mu}=Y \partial^{\mu} Y^{\dagger}+g^{\mu}
$$


Therefore the lagrangian becomes

$$
\begin{aligned}
\mathcal{L} & =-\frac{F^{2}}{4} \operatorname{Tr}\left[\left(X \partial^{\mu} X^{\dagger}-Y \partial^{\mu} Y^{\dagger}\right)^{2}\right]-\alpha \frac{F^{2}}{4} \operatorname{Tr}\left[\left(X \partial^{\mu} X^{\dagger}+Y \partial^{\mu} Y^{\dagger}+2 g_{\mu}\right)^{2}\right] \\
& -\frac{f^{2}}{2}\left(J_{\phi}^{\mu}\right)^{2}+\text { kinetic term for } g^{\mu}
\end{aligned}
$$

Defining

$$
g_{\mu}=i g_{s} \frac{T_{a}}{2} g_{\mu}^{a}
$$

where $g_{s}$ is the QCD coupling constant, we see that the gluon field acquires a mass (more discussion later). In the limit where the Goldstones have energies much smaller than the gluon mass, we can neglect the kinetic term for the gluon. Then the lagrangian (21) is nothing but the hidden gauge symmetry version of the chiral lagrangian for QCD [7] (except for the contribution of the field $\phi$ ). In fact, in this limit, the gluon field becomes an auxiliary field which can be eliminated through its equation of motion

$$
g_{\mu}=-\frac{1}{2}\left(X \partial_{\mu} X^{\dagger}+Y \partial_{\mu} Y^{\dagger}\right)
$$

obtaining

$$
\mathcal{L}=-\frac{F^{2}}{4} \operatorname{Tr}\left[\left(X \partial_{\mu} X^{\dagger}-Y \partial_{\mu} Y^{\dagger}\right)^{2}\right]-\frac{f^{2}}{2}\left(J_{\phi}^{\mu}\right)^{2}=\frac{F^{2}}{4} \operatorname{Tr}\left[\partial_{\mu} \Sigma \partial^{\mu} \Sigma^{\dagger}\right]-\frac{f^{2}}{2}\left(J_{\phi}^{\mu}\right)^{2}
$$

where

$$
\Sigma=Y^{\dagger} X
$$

transforms under the group $S U(3)_{c} \otimes S U(3)_{L} \otimes S U(3)_{R}$ as

$$
\Sigma \rightarrow g_{R}^{*} \Sigma g_{L}^{T}
$$

The field $\Sigma^{T}$ transforms exactly as the standard chiral field of the non linearrealization of QCD (in the chiral broken phase).

The preceding discussion applies to any situation for which the mass of the gluon is at least larger of the typical gap parameter. For a situation in which the mass of the gluon is much smaller than the gap parameter the degrees of freedom of the massive gluons have to kept in the effective 
lagrangian. For such a situation we can make use of the gauge freedom to choose a gauge such that $X=Y^{\dagger}$. This implies

$$
\tilde{\Pi}_{X}=-\tilde{\Pi}_{Y}
$$

or

$$
\Pi_{X}=0, \quad \Pi_{Y}=F \tilde{\Pi}_{X}
$$

This gauge is the unitary gauge. In fact the bilinear term in the Goldstones and in the gluon field in eq. (21) is proportional to

$$
g^{\mu} \partial_{\mu}\left(\tilde{\Pi}_{X}+\tilde{\Pi}_{Y}\right)
$$

and therefore cancels out. It follows that the gluon mass is given by

$$
m_{g}^{2}=\alpha g_{s}^{2} \frac{F^{2}}{4}
$$

Notice also that the $X \leftrightarrow Y$ symmetry, in this gauge, implies $\Pi_{Y} \leftrightarrow-\Pi_{Y}$.

The identification with the low-energy effective lagrangian of QCD could not seem to be really correct, since in this phase we are breaking Lorentz invariance. However in the low-energy limit, where only the fields $\Sigma$ and $\phi$ are present, this effect amounts to take as lagrangian

$$
\mathcal{L}=\frac{F_{T}^{2}}{4}\left(\operatorname{Tr}\left[\dot{\Sigma} \dot{\Sigma}^{\dagger}\right]-v_{Y}^{2} \operatorname{Tr}\left[\partial^{i} \Sigma \partial^{i} \Sigma^{\dagger}\right]\right)-\frac{f_{T}^{2}}{2}\left(\left(J_{\phi}^{0}\right)^{2}-v_{\phi}^{2}\left(J_{\phi}^{i}\right)^{2}\right)
$$

We see that by a rescaling of the coordinates $x^{i} \rightarrow v_{Y} x_{i}$ (that is by using a coordinate system with the $v_{Y}=1$ ), the first term becomes exactly the chiral lagrangian for QCD.

The question remains of the Goldstone $\phi$ associated to the breaking of the baryon number. According to ref. [3] this could be interpreted as a dibaryon state, the $H$, a spin $0 S U(3)$ singlet of composition (udsuds). In fact, it had been pointed out by R. Jaffe [8], that this (deus ex machina) state is particularly light.

\section{Gauging $U(1)_{\mathrm{em}}$}

The gauging of $U(1)_{\mathrm{em}}$ (formal extension to the full electroweak group is straightforward) is made through covariant derivatives

$$
D_{\mu} X=\partial_{\mu} X-g_{\mu} X-X Q A_{\mu}, \quad D_{\mu} Y=\partial_{\mu} Y-g_{\mu} Y-Y Q A_{\mu}
$$


where $Q=\operatorname{diag}(+2 / 3,-1 / 3,-1 / 3$,$) . We see immediately that the com-$ bination of $Q$ and of the generator of $S U(3)_{c}$ proportional to $Q_{S U(3)_{c}}=$ $\operatorname{diag}(-2 / 3,+1 / 3,+1 / 3)=-Q$, leaves invariant the ground state. In fact,

$$
Q_{S U(3)_{c}}\langle X\rangle+\langle X\rangle Q \rightarrow\left(Q_{S U(3)_{c}}\right)_{a b} \delta_{b i}+\delta_{a i} Q_{i j}=0
$$

It follows that one combination of $g^{Q}$ (the gluon field associated to $Q_{S U(3)_{c}}$ ) and $A_{\mu}$ is massive, whereas the orthogonal combination remains massless. Since the component of the gluon field involved in the mixing, with the appropriate normalization, is given by

$$
g_{\mu} \rightarrow \frac{i}{2} \sqrt{\frac{3}{2}} g_{s} g_{\mu}^{Q} Q
$$

and $A_{\mu}$ is defined as

$$
A_{\mu} \rightarrow i e A_{\mu}
$$

one easily finds that the massless combination is

$$
A^{\prime}=A \cos \psi+g_{Q} \sin \psi
$$

with

$$
\tan \psi=\sqrt{\frac{8}{3}} \frac{e}{g_{s}}
$$

As a last comment, let us consider at this order in the derivatives the WZW term associated to the $U(1)$ anomaly. It will be proportional to

$$
\operatorname{Tr}\left(\tilde{Q}^{2} \Pi_{Y}\right) \epsilon_{\mu \nu \rho \sigma} F^{\mu \nu} F^{\rho \sigma}
$$

The calculation of the trace can be done by evaluating the contribution of the quarks contributing in the loop, by using the definition of the electric charge in this basis. In an equivalent way we can use the previous expression, being careful that the action of the electric charge in this phase is really of the type

$$
\tilde{Q}=1 \otimes Q-Q \otimes 1
$$

where $Q=\operatorname{diag}(+2 / 3,-1 / 3,-13)$ and the tensor product refers to the color and flavor indices respectively. Then, by taking the trace with the $\Pi_{Y}$ field (acting on the flavor space since it comes from $\Sigma$ ), we get easily

$$
\operatorname{Tr}\left[\tilde{Q}^{2} \Pi_{Y}\right]=3 \operatorname{Tr}\left[Q^{2} \Pi_{Y}\right]
$$


Therefore, also in this phase we get the anomalous decay of the two mesons lying in the center of $S U(3)$, since the previous trace gets contribution both from $T_{3}$ and from $T_{8}$, as it can be seen by using the expression of $Q$ in terms of the $S U(3)$ generators

$$
Q=\frac{\sqrt{3}}{2}\left(T_{3}+\frac{1}{\sqrt{3}} T_{8}\right)
$$

This calculation gets much simpler by using a basis of flavor where the indices $i=1,2,3$ correspond to $s, d, u$ respectively, since in this basis $Q$ is proportional to $T_{8}$.

\section{Conclusions}

We have shown that QCD for three massless flavors in the color-flavor locked (CFL) phase at high densities is described at low energies by an effective lagrangian which is the same as the low energy effective lagrangian of the chiral phase. The gluons become massive in the CFL phase and the effective lagrangian of such a phase, when reinterpreted in the chiral phase, describes an octect of vectors coupled in a chiral invariant way, allowing for the interpretation of the massive gluons as corresponding to the ordinary vector

bosons. The lack of Lorentz invariance in the dense phase does not affect the argument.

\section{Acknowledgements}

R.C. would like to thank the Theory Division of CERN for the kind hospitality offered to him during the final stage of this paper.

\section{References}

[1] F. Barrois, Nucl. Phys. B129, 390 (1977); S.C.Frautschi, in Workshop on hadronic matter at extreme densities, Erice 1978.

[2] M. Alford, K. Rajagopal and F. Wilczek, Nucl.Phys. B537 (1999) 443. 
[3] T. Schäffer and F. Wilczek, Phys. Rev. Lett. 82 (1999) 3956; ibidem hepph/9903503; M. Alford, J. Berges, and K. Rajagopal, hep-ph/9903502.

[4] D. Bailin and A. Love, Phys. Rep. 107, 325 (1984); M. Alford, K. Rajagopal and F. Wilczek, Phys.Lett. B422 (1998) 247; R. Rapp, T. Schäffer, E.V. Shuryak and M. Velkovsky, Phys. Rev. Lett. 81 (1998) 53; J. Berges and K. Rajagopal, Nucl. Phys. B538, 215 (1999); T. Schäfer and F. Wilczek, Phys. Lett. B450, 325 (1999); D.T. Son, Phys. Rev. D 59, 094019 (1999). N. Evans, S.D.H. Hsu, and M. Schwetz, Nucl. Phys. B551, 275 (1999); G.W. Carter and D. Diakonov, Phys. Rev. D 60, 016004 (1999); N. Evans, S. Hsu, M. Schwetz, hep-ph/9810514; R.D. Pisarski and D.H. Rischke, nucl-th/9811104; K. Langfeld and M. Rho, hep-ph/9811227; J. Berges, D-U Jungnickel, and C.Wetterich, hepph/9811387; D.K. Hong, hep-ph/9812510; N.O. Agasian, B.O. Kerbikov, and V.I. Shevchenko, hep-ph/9902335; R.D. Pisarski and D.H. Rischke, nucl-th/9903023; T.M. Schwarz, S.P. Klevansky, and G.Papp, nucl-th/9903048; D.G. Caldi and A. Chodos, hep-ph/9903416; R. Rapp, T. Schäfer, E.V. Shuryak, and M. Velkovsky, hep-ph/9904353; E. Shuster and D.T. Son, hep-ph/9905448; D.K. Hong, hep-ph/9905523; R.D. Pisarski and D.H. Rischke, nucl-th/9906050; D.K. Hong, V.A. Miransky, I.A. Shovkovy, and L.C.R. Wijewardhana, hep-ph/9906478; T. Schäfer and F. Wilczek, hep-ph/9906512; R.D. Pisarski and D.H. Rischke, nuclth/9907041.

[5] J. Wess and B. Zumino, Phys. Lett. B37 (1971) 95; E. Witten, Nucl. Phys. B223 (1983) 433.

[6] D. K. Hong, M. Rho and I. Zahed, hep-ph/9906551.

[7] A.P. Balachandran, A. Stern, and G. Trahern, Phys. Rev. D19 (1979) 2416; M. Bando, T. Kugo and K. Yamawaki, Phys. Rep. 164 (1988) 217; R. Casalbuoni et al., Phys. Lett. 292B (1992) 371.

[8] R. Jaffe, Phys. Rev. Lett. 38 (1977) 195, 617(E). 